\title{
The search for optimal response prediction in cancer leads to a personalized approach
}

\author{
Eric Van Cutsem • Fortunato Ciardiello
}

Received: 22 March 2010 /Accepted: 22 March 2010 /Published online: 28 March 2010

(C) Springer-Verlag 2010

Keywords Response prediction - Molecular markers .

Functional imaging · Targeted therapy

There is a clear need for better response prediction in the management of patients with cancer. Important progress has indeed been made in understanding the underlying mechanisms of tumor growth and targeting important new targets with novel drugs blocking receptors or interfering with one of the important pathways. A long list of new, targeted agents has been developed or is under development. With this comes also the need of new methods and new molecular markers for response prediction.

The area of response prediction is booming: functional imaging is becoming a very important tool and aid and the knowledge on molecular markers is growing very rapidly. Functional imaging with positron emission tomography (PET) scan, dynamic contrast-enhanced ultrasonography (DCE-US) and magnetic resonance imaging (MRI) is coming into the research arena and is likely to be implemented in the future in clinical practice. Better validation of the clinical utility of functional imaging techniques is, however, still needed in many clinical situations. A major clinical problem for the use of novel molecular targeted agents is to determine their activity by the use of classic imaging evaluation methods such as

E. Van Cutsem $(\bowtie)$

University Hospital Gasthuisberg,

Leuven, Belgium

e-mail: eric.vancutsem@uz.kuleuven.ac.be

F. Ciardiello

Second University of Naples,

Naples, Italy computed tomography (CT) and by the use of standard tumor size assessment with Response Evaluation Criteria in Solid Tumors (RECIST). Therefore, functional imaging techniques that could be performed in the evaluation of the early activity and also in the determination of the optimal dose of the new targeted agents are necessary. Dynamic contrast-enhanced (DCE)-MRI and DCE-US, diffusion and perfusion MRI and PET-CT, using new tracers for proliferation, will probably be integrated gradually in the new generation of clinical trials and eventually also in clinical practice.

Molecular markers clearly have the potential to help to select cancer patients that are suitable for treatment with a molecular targeted, agent if they are able to predict the activity or the lack of activity of these agents. Among examples of predictive biomarkers for response and clinical activity, there are cKIT mutations for imatinib therapy in gastrointestinal stromal tumors (GIST), epidermal growth factor receptor (EGFR) mutations for erlotinib and gefitinib treatment in non- small cell lung cancer (NSCLC), HER-2 gene amplification for the use of trastuzumab in breast cancer, as well as recently HER-2 overexpression in gastric cancer, KRAS mutations as predictors of lack of activity of cetuximab and panitumumab in colorectal cancer and, finally, BRAF mutations for treatment with selective BRAF inhibitors in melanoma. This list is not completed and will certainly be expanded in the near future. Further, some of these biomarkers are not only predictive for response to a given treatment, but they could have also a prognostic role. As an example, BRAF mutations correlate with poor prognosis, lack of cetuximab and panitumumab efficacy and poor response to chemotherapy in metastatic colorectal cancer. The discovery of whole gene signatures, if they would be confirmed predictive and/or prognostic, will for 
sure have major implications in the clinical management of molecular targeted treatments of cancer patients.

Another example that illustrates these rapid developments, but also highlights the difficulties is the finding that KRAS plays a central role in the process of colorectal tumor growth. Through this finding, the unmet need of new therapeutic strategies for KRAS mutant tumors became clear as they do not respond to EGFR targeted agents. Blocking a single downstream target of RAS is theoretically an interesting approach, but will probably not be sufficient to result in inhibition of tumor growth as RAS signals through very complex branching pathways. Therefore, a dual-targeted or multi-targeted therapeutic strategy may be more efficient, but this still needs to be confirmed in clinical trials, which are currently ongoing.

It is, therefore, very important to include mechanismbased translational research approaches in the early clinical trials of novel molecular targeted drugs for the discovery and the evaluation of potential biomarkers, which subsequently can be validated prospectively with the integration of functional imaging. In this respect, molecular biomarkers and functional imaging techniques should have a complementary role for improving therapy outcome, by optimizing a truly personalized treatment approach for our cancer patients, that deserve it in the new era of molecular targeted cancer therapies. 